\title{
Propuesta de una herramienta de análisis de contenido para el emplazamiento de producto en contenidos audiovisuales
}

\author{
José MARTÍ PARREÑO ${ }^{1}$ \\ Universitat de València
}

\begin{abstract}
RESUMEN:
El emplazamiento de producto ha ido ganando terreno en los últimos años como parte de las comunicaciones de marketing de los anunciantes. Esta herramienta de comunicación ha ido evolucionando rápidamente en el entorno mediático actual transformándose en una herramienta mucho más compleja con la que alcanzar diferentes objetivos de marketing. Este interés en la praxis comercial ha ido acompañando por un creciente interés académico sobre el emplazamiento de producto. Sin embargo, en la actualidad todavía se carece de una herramienta ampliamente aceptada que permita identificar las diversas categorías de emplazamiento de producto que pueden ser detectadas en una amplia variedad de contenidos audiovisuales. El presente trabajo tiene como objetivo principal el desarrollo de una herramienta de análisis que utilice como metodología el análisis de contenido.
\end{abstract}

PALABRAS CLAVE: emplazamiento de producto; análisis de contenido; metodología; comunicaciones de marketing; herramientas de investigación.

TITLE: A Proposal of a Tool for Content Analysis of Product Placement in Audiovisual Content.

\begin{abstract}
:
Product placement is gaining momentum within advertisers' marketing communications. This communication tool has been quickly evolving in nowadays media environment and has become a much more complex tool used to achieve different marketing goals. This interest in the market has also increase the academic interest about product placement. Despite this growing interest there is a lack of a tool broadly accepted that allow researchers to identify the different categories of product placement that can be detected in a broad range of audiovisual content. This paper has as a main objective the development of such an analysis tool using a content analysis methodology.
\end{abstract}

KEY WORDS: product placement; content analysis; methodology; marketing communications; research tools.

\section{Introducción}

El emplazamiento de producto ha sido utilizado como herramienta de las comunicaciones de marketing de las organizaciones desde el nacimiento mismo de los

1 Profesor del Departamento de Comercialización e Investigación de Mercados. Facultad de Economía. Universitat de València. E-mail: jose.marti-parreno@uv.es 
medios de comunicación de masas ${ }^{2}$. En el cine comercial, por ejemplo, esta práctica se produce de forma habitual a partir de los años $20^{3}$. En la radio, la presencia de marcas, productos y patrocinadores permitió el desarrollo de géneros como la radionovela ${ }^{4}$. En el medio televisivo el emplazamiento de producto es habitual no sólo en las denominadas comedias de situación ${ }^{5}$ sino en la programación general ${ }^{6}$. También en los videojuegos se ha producido un importante aumento de la presencia de marcas y productos en los últimos años ${ }^{7}$. La saturación publicitaria y, por tanto, el hastío del consumidor hacia ella, mostrado mediante comportamientos como el zapping; la aparición de nuevos dispositivos como los DVR (Digital Video Recorders), que permiten saltar o incluso borrar los anuncios; y una creciente convergencia entre los contenidos publicitarios y los contenidos de entretenimiento ${ }^{8}$ han sido señalados como algunos de los factores principales por los cuales las herramientas publicitarias clásicas han ido perdiendo eficacia, motivando una reorientación de los presupuestos destinados a comunicaciones de marketing hacia los denominados medios alternativos como el emplazamiento de producto ${ }^{9}$. Esta tendencia queda bien reflejada en el crecimiento de la inversión en emplazamiento de producto durante los últimos años. Exceptuando el año 2009 (en el que se produjo un descenso del $2.8 \%$ de la inversión en emplazamiento de producto pagado en los EE.UU motivado por los recortes presupuestarios derivados de la crisis económica) la inversión había crecido hasta el $27.1 \%$ en el período $2004-2009^{10}$. La tendencia al alza de la inversión en emplazamiento de producto queda reflejada por el hecho de que hasta el 2014 se espera un incremento a nivel mundial de en torno al 9.1\% en el denominado branded entertainment ${ }^{11}$, siendo el emplazamiento de producto

2 Newell, J.; SAlmon, C. T.; Chang, S. (2006): «The Hidden History of Product Placement», Journal of Broadcasting and Electronic Media, 50 (4), 575-594.

3 Galician, M. L.; Bourdeau, P. G. (2004): «The Evolution of Product Placements in Hollywood Cinema: Embedding High-Involvement 'Heroic' Brand Images», Journal of Promotion Management, 10 (1/2), 15-36.

Allen, R. C. (1985): Speaking of Soap Operas, Nueva York, Schoken Books.

5 Russell, C. A.; STERN, B. B. (2006): «Consumers, Characters, and Products: A Balance Model of Sitcom Product Placement Effects», Journal of Advertising, 35 (1), 7-21.

6 AVERY, R. J.; FERRARO, R. (2000): «Verismilitude or advertising? Brand appearances on primetime televisión», The Journal of Consumer Affairs, 34 (2), 217-244.

7 TAPIA, A.; LÓPEZ, M.; GonZÁlez, P. (2009): «Publicidad in-Game. Los videojuegos como soporte publicitario», Pensar la Publicidad. Revista Internacional de Investigaciones Publicitarias, 3 (2), 73-88.

8 Donaton, S. (2004): Madison \& Vine: Why the Entertainment and Advertising Industries Must Converge to Survive, Nueva York, McGraw-Hill.

9 MARTí, J.; MuÑOZ, P. (2008): Engagement Marketing: Una nueva publicidad para un marketing de compromiso, Madrid, Financial Times/Prentice Hall.

10 PQ Media (2010): Global Branded Entertainment Marketing Forecast 2010-2014. En http://www.pqmedia.com/brandedentertainmentforecast2010.html. Web visitada el: 07/08/10.

11 LEHU, J. M. (2007): Branded entertainment: product placement and brand strategy in the entertainment business, Londres y Filadelfia, Kogan Page. 
el segmento de mayor crecimiento ${ }^{12}$. Este incremento del emplazamiento de producto en la praxis comercial ha ido acompañando por un creciente interés académico $^{13}$ del estudio de los emplazamientos en diversos contenidos audiovisuales que abarcan desde los largometrajes hasta las comedias de situación o los videojuegos. Sin embargo, la mayoría de estos estudios han tenido como objetivo medir los efectos de los emplazamientos sobre las audiencias en relación a métricas de eficacia publicitaria como el recuerdo y reconocimiento de marca; la actitud hacia la marca; o la intención de compra. Así en el cine comercial, se han medido los efectos generados por los emplazamientos sobre las audiencias en relación a: recuerdo y reconocimiento de la marca o producto emplazado ${ }^{14}$; actitud hacia el emplazamiento ${ }^{15}$; actitud hacia la marca o producto emplazado ${ }^{16}$; intención de compra ${ }^{17}$; influencia

12

13 BAÑos, M.; RodRÍGUEZ, T. (2003): Product placement: estrella invitada la marca, Madrid, Cie Inversiones Editoriales Dossat 2000; MÉNDIZ, A. (2000): Nuevas Formas Publicitarias: Patrocino, Product Placement y Publicidad en Internet, Málaga, Servicio de Publicaciones de la Universidad de Málaga; VICTORIA, J. S. (1999): Presencia, comunicación y estrategia de las marcas comerciales en la ficción audiovisual, Universidad de Málaga, Tesis doctoral; DeL PINO, C. (2004): Marcas y ficción televisiva: el product placement en las teleseries españolas (1991-2002), Universidad de Málaga, Tesis doctoral.

14

Delorme, D. E.; ReID, L. N.; ZimMER, M. R. (1994): «Brands in Films: Young Moviegoers' Experiences and Interpretations», en KING, K. W. (ed.), Proceedings of the 1994 Conference of the American Academy of Advertising. Athens (Georgia), American Academy of Advertising, 60. SABHERwaL, S., POKRYWCZYNSKI, J.; GRIFFIN, R. (1994): «Brand Recall for Product Placements in Motion Pictures: A Memory-Based Perspective», paper presentado en la Conference of the Association for Education in Journalism and Mass Communication, Atlanta (Georgia). BABIN, L. A.; CARDER, S.T. (1996): «Viewers' Recognition of Brands Placed within a Film» International Journal of Advertising, 15 (2), 140-151. GuPTA, P. B.; LORD, K. R. (1998): «Product Placement in Movies. The Effect of Prominence and Mode on Audience Recall», Journal of Current Issues and Research in Advertising, 20 (1), 47-59. BRENNAN, I.; DuBAS K. M.; BABIN L. A. (1999): «The Influence of Product-Placement Type and Exposure Time on Product-Placement Recognition» International Journal of Advertising, 18 (3), 323-337. D'Astous, A.; ChARTIER, F. (2000): «A Study of Factors Affecting Consumer Evaluations and Memory of Product Placements in Movies», Journal of Current Issues and Research in Advertising, 22 (2), 31-40.

15 NeBenZAHL, I. D.; SECUNDA, E. (1993): «Consumers' attitudes toward product placement in movies», International Journal of Advertising, 12 (1), 1-11. GUPTA, P. B. Y GouLD, S. J. (1997): «Consumer's Perceptions of the Ethics and Acceptability of Product Placement in Movies: Product Category and Individual Differences», Journal of Current Issues and Research in Advertising, 19 (1), 38-50. GoulD, S. J., GuPTA, P. B.; GrABNER-KRÄUTER, S. (2000): «Product Placements in Movies: A Cross-Cultural Analysis of Austrian, French and American Consumer's Attitudes Toward This Emerging International Promotion Medium», Journal of Advertising, 29 (4), 41-58. D'Astous, A.; CHARTIER, F. (2000): «A Study of Factors Affecting Consumer Evaluations and Memory of Product Placements in Movies», Journal of Current Issues and Research in Advertising, 22 (2), 31-40. MCKECHNIE, S. A.; ZHOU, J. (2003): «Product Placement In Movies: A Comparison Of Chinese And American Consumers' Attitudes», International Journal of Advertising, 22, 349-374.

16 HowARD, D. L.; BARRY, T. E. (1994): «The Role of Thematic Congruence Between a MoodInduced Event and an Advertised Product in Determining the Effects of Mood on Brand Attitudes», Journal of Consumer Psychology, 3 (1), 1-27.

17 Goldberg, M. E.; Gorn, G. J. (1987): «Happy and Sad TV Programs: How They Affect Reactions to Commercials», Journal of Consumer Research, 14 (diciembre), 387-403. 
sobre la elección de marca $^{18}$. Existe sin embargo una carencia de herramientas que permitan identificar de manera sistemática, y ampliamente aceptada, los emplazamientos de producto aparecidos en contenidos audiovisuales.

El presente trabajo tiene como objetivo principal el desarrollo de una herramienta de análisis que, utilizando el análisis de contenido como metodología de trabajo, permita identificar y tipificar las diversas categorías de emplazamiento de producto que pueden ser detectadas en una amplia variedad de contenidos audiovisuales. Para ello, primeramente se conceptualizará el emplazamiento de producto como herramienta de las comunicaciones de marketing. A continuación se definirá lo que se entiende por contenido audiovisual como soporte de esta herramienta. Posteriormente se describirá el análisis de contenido como herramienta de análisis y su aplicación a la detección del emplazamiento de producto en los contenidos audiovisuales. Tras este marco conceptual, se procederá a la exposición de la metodología utilizada para desarrollar esta herramienta de análisis y finalmente se presentará la herramienta obtenida. Las implicaciones para académicos y profesionales del marketing serán asimismo expuestas al igual que las conclusiones, las limitaciones del estudio y las futuras líneas de investigación de interés.

\section{El emplazamiento de producto}

El emplazamiento de producto o product placement ha sido definido como «la práctica de incluir el nombre, producto, embalaje, señalización u otro artículo de merchandising de una marca, en una película de cine, en televisión o en otros medios con el fin de incrementar la notoriedad y el reconocimiento inmediato de la marca en el punto de compra ${ }^{19}$. Esta herramienta de las comunicaciones de marketing se enmarca en los denominados mensajes híbridos, es decir, «todo intento pagado de influir a las audiencias para obtener un beneficio comercial usando comunicaciones que presentan un carácter no comercial» ${ }^{20}$. En este sentido, los mensajes híbridos gozan de las ventajas de dos de las principales herramientas de comunicación no personal utilizadas tradicionalmente por las organizaciones: la publicidad y la publicidad gratuita. Por una parte, estos mensajes son pagados por la organización, lo que permite un control sobre los mismos (publicidad); por otra, la organización no es identificada claramente como emisor del mensaje, por lo que

18 AutY, S.; LEwIS, C. (2004): «Exploring Children's Choice: The Reminder Effect of Product Placement», Psychology and Marketing, 21 (9), 697-708.

19 PANDA, T. K. (2004): «Effectiveness of Product Placements in Indian Films and its effects on Brand Memory and Attitude with Special Reference to Hindi Films», The ICFAI Journal of Marketing Management, (agosto), 42.

20 Balasubramanian, S. K. (1994): «Beyond Advertising and Publicity: Hybrid Messages and Public Policy Issues», Journal of Advertising, 23 (4), 30. 
su aparente falta de intención comercial (publicidad gratuita) puede redundar en una mayor credibilidad del mensaje por parte del receptor ${ }^{21}$.

Entre las ventajas que aporta el emplazamiento de producto a las comunicaciones de marketing de las organizaciones está la aproximación indirecta a los consumidores. Puesto que el mensaje no se comunica explícitamente a través de un anuncio sino que es más bien potenciado por el contexto (película, serie de televisión, videojuego, etc.) puede generar menos respuestas cognitivas desfavorables que una aproximación directa ${ }^{22}$. Asimismo, puesto que el foco de atención principal es el contenido de entretenimiento, el foco secundario (la comunicación de marca) se procesa más implícitamente, pudiendo evitarse en mayor grado procesos como la contrargumentación del consumidor al mensaje. Este hecho está en consonancia con la ruta periférica hacia la persuasión ${ }^{23}$ ya que se ha detectado que los emplazamientos naturales no despiertan la contrargumentación y es más posible que produzcan efectos afectivos positivos ${ }^{24}$. Así por ejemplo, en el caso de los videojuegos, se ha detectado que el emplazamiento de marcas comerciales es aceptado positivamente por los videojugadores si se trata de marcas reales que aportan realismo al videojuego ${ }^{25}$. También se ha detectado que los espectadores cinematográficos aceptan positivamente los emplazamientos de producto $^{26}$ especialmente cuando se trata de marcas familiares que aportan realismo y ayudan a caracterizar a los personajes ${ }^{27}$. Otra de las ventajas de esta herramienta de comunicación es que, puesto que la marca o producto emplazado forman parte del contenido de entretenimiento, se evitan comportamientos del consumidor como el zapping por parte de estas audiencias cautivas ${ }^{28}$.

21 Ibídem.

22 YI, Y. (1990): The Effects of Contextual Priming in Print Advertisements, Journal of Consumer Research, 17 (2), 215-222.

23 PetTy, R. E.; CACIOPPO, J. T.; SCHUMAnN, D. (1983): «Central and Peripheral Routes to Advertising Effectiveness: The Moderating Role of Involvement», Journal of Consumer Research, 10 (2), 135-146. PETTY, R. E.; CACIOPPO, J. T. (1986): «The Elaboration Likelihood Model of Persuasion», en Berkowitz, L. (ed.). Advances in Experimental Social Psychology, vol. 19, Nueva York, Academia Press, 123-205.

24 D'Astous, A.; Chartier, F. (2000): «A Study of Factors Affecting Consumer Evaluations and Memory of Product Placements in Movies», Journal of Current Issues and Research in Advertising, 22 (2), $31-40$.

25 Nelson, M. R. (2002): «Recall of Brand Placements in Computer/Video Games», Journal of Advertising Research, 42 (2), 80-92. Nelson, M. R.; KeUM, H.; YAROS, R. A. (2004): «Advertainment or Adcreep? Game Players' Attitudes toward Advertising and Product Placements in Computer Games», Journal of Interactive Advertising, 5 (1), http://www.jiad.org/vol5/no1/nelson/ Web visitada el: 18/06/06. MOLESWORTH, M. (2006): «Real Brands in Imaginary Worlds: Investigating Players'Experiences of Brand Placement in Digital Games», Journal of Consumer Behaviour, 5 (4), 355-366.

26 SChMOLl, N.M.; HAFER, J.; Hilt, M.; ReILly, H. (2006): «Baby boomers’ attitudes towards product placements», Journal of Current Issues and research in Advertising. 2 (8), 33-53.

27 Delorme, D. E.; ReID, L. N.; Zimmer, M. R. (1994): «Brands in Films: Young Moviegoers' Experiences and Interpertations», en KING, K. W. (ed.), Proceedings of the 1994 Conference of the American Academy of Advertising. Athens (Georgia), American Academy of Advertising, 60.

28 Troup, M. M. (1991): The Captive Audience: A Content Analysis Of Product Placements In Motion Pictures, Tesis doctoral, Tallahassee, Florida State University. 


\section{Definición de contenido audiovisual}

La presente herramienta de análisis ha sido desarrollada pensando en la identificación y tipificación de emplazamientos de producto en contenidos audiovisuales. Por contenido audiovisual se entiende aquí cualquier género (de entretenimiento o informativo) que utilice un lenguaje audiovisual para difundir su mensaje y que sea consumido por la audiencia a través de cualquier medio audiovisual (televisión, Internet, telefonía móvil, etc.). Esta conceptualización amplia considera contenido audiovisual desde un largometraje cinematográfico hasta una serie de televisión, un informativo, un reality show, un documental o un videojuego.

\section{El análisis de contenido}

El análisis de contenido ${ }^{29}$ es una herramienta de análisis consistente en «una técnica de investigación por medio de la descripción objetiva, sistemática y cuantitativa del contenido manifiesto de un comunicador ${ }^{30}$. Entre las ventajas que ofrece esta herramienta se encuentra la uniformidad del análisis efectuado por diferentes investigadores, la disminución de los juicios personales y subjetivos en el procesamiento de la información y el análisis de los contenidos tanto latentes como manifiestos en base a unos criterios objetivamente definidos por los jueces que efectúan el análisis ${ }^{31}$. Este análisis permite no sólo la transformación de la información en datos que pueden ser resumidos y comparados ${ }^{32}$ sino que permitan extraer información que debe estar vinculada a otra información con la que explicar algún hecho ${ }^{33}$.

Un paso previo a la realización de cualquier análisis de contenido viene determinado por las reglas de codificación en base a una determinada muestra, unas unidades de análisis y unas categorías de análisis ${ }^{34}$. Estas categorías de análisis deben ser bien definidas para alcanzar una fiabilidad aceptable tanto en la codificación de las unidades de análisis como en los resultados obtenidos por diferentes jueces con la finalidad de garantizar la validez del instrumento de análisis ${ }^{35}$. Por

29

KASSARJIAN, H. H. (1977): «Content Analysis in Consumer Research», Journal of Consumer Research, 4 (1), 8-19. BARDIN, L. (1977): El análisis de contenido, Madrid, Akal. KRIPPENDORF, K. (1980): Content analysis: and introduction to its methodology, Beverly Hills, Sage Publications, 46-51.

30 Berelson, B. (1952): Content Analysis in Communications Research, Glencoe (Illinois), The Free Press, 55.

31 FeARING, F. (1953): «Towards a Psychological Theory of Human Communication», Journal of Personality, 22, 71-88.

32 PAisley, W. J. (1969): «Studying Style as Deviation of Encoding Norm», en GERBNER, G. et alt. (eds.), The Analysis of Communications Content: Developments in Scientific Theories and Computer Techniques, Nueva York, Willey, 133-146.

\footnotetext{
33 BERELSON, op. cit.

34 KASSARJIAN, op cit.

35 Ibídem.
} 
tanto la adecuada elaboración del instrumento de medida (normalmente una ficha de análisis) resulta fundamental para el éxito del análisis efectuado. El análisis de contenido cuenta con una larga tradición como herramienta de análisis de los mensajes emitidos por los medios de comunicación y ya ha sido aplicado con éxito al estudio del emplazamiento de producto ${ }^{36}$.

\section{Metodología}

Con el fin de elaborar la herramienta de análisis se procedió a una exhaustiva revisión de la literatura académica sobre el emplazamiento de producto. Esta revisión incluyó no sólo trabajos sobre el cine o la televisión sino también sobre el emplazamiento de producto en videojuegos. El objetivo fundamental de esta revisión fue la identificación de las principales variables que han sido estudiadas en trabajos empíricos en relación al emplazamiento de producto en contenidos audiovisuales. Un total de 62 trabajos sobre el emplazamiento de producto en el cine, la televisión y los videojuegos fueron seleccionados como muestra de estudio. Aquellos trabajos no relacionados con las variables de interés para el estudio (por ejemplo, trabajos sobre la percepción del emplazamiento de producto por parte de los anunciantes o los consumidores; trabajos que abordaban aspectos legales de la técnica o intención de uso por parte de los anunciantes, etc.) fueron descartados. La muestra final de análisis quedó compuesta por un total de 51 trabajos sobre el emplazamiento de producto en el cine, la televisión y los videojuegos. De ellos, 24 analizaban los efectos del emplazamiento de producto en el medio cinematográfico; 9 analizaban los efectos del emplazamiento de producto en televisión; y 18 analizaban los efectos del emplazamiento de producto en los videojuegos. La muestra analiza está recogida en las Tablas 1, 2 y 3.

Tabla 1. Estudios analizados sobre emplazamiento de producto en cine.

\begin{tabular}{|c|c|c|c|}
\hline TÍTULO & AUTOR & $\boldsymbol{A N} \boldsymbol{O} O$ & VARIABLES \\
\hline $\begin{array}{l}\text { The Cost Effiency And Commu- } \\
\text { nication Effects Associates With } \\
\text { Brand Name Exposure Within } \\
\text { Motion Pictures }\end{array}$ & Steortz & 1987 & $\begin{array}{l}\text { Ubicación del emplazamiento, moda- } \\
\text { lidad del emplazamiento (ver- } \\
\text { bal/visual) e interacción de los perso- } \\
\text { najes con las marcas. }\end{array}$ \\
\hline
\end{tabular}

36 Troup, M. M. (1991): The Captive Audience: A Content Analysis Of Product Placements In Motion Pictures, Tesis doctoral, Tallahassee, Florida State University. SAPOLSKY, B. S.; KINNEY, L. (1994): «You Oughta Be in Pictures: Product Placements in the Top Grossing Films of 1991», en KING, K. W. (ed.). Proceedings of the 1994 Conference of the American Academy of Advertising. Athens (Georgia), American Academy of Advertising, 89. Moore, E. S. (2006): «It's Child Play: Advergaming and the Online Marketing of Food to Children», The Henry J. Kaiser Family Foundation, http://www.kff.org/entmedia/upload/It-s-Child-s-Play-Advergaming-and-the-Online-Marketing-of-Foodto-Children-Bios.pdf Web visitada el: 28/06/07. 


\begin{tabular}{|c|c|c|c|}
\hline TÍTULO & AUTOR & $A \tilde{N} O$ & VARIABLES \\
\hline $\begin{array}{l}\text { The Captive Audience: A Content } \\
\text { Analysis Of Product Placements } \\
\text { In Motion Pictures }\end{array}$ & Troup & 1991 & $\begin{array}{l}\text { Ubicación, contexto, interacción de } \\
\text { los personajes con las marcas, caracte- } \\
\text { rísticas del emplazamiento, tipo de } \\
\text { personaje, encuadre, duración. }\end{array}$ \\
\hline $\begin{array}{l}\text { Consumers' attitudes toward } \\
\text { product placement in movies }\end{array}$ & Nebenzahl y Secunda & 1993 & $\begin{array}{l}\text { Exposición a las marcas emplazadas } \\
\text { en un largometraje. }\end{array}$ \\
\hline $\begin{array}{l}\text { Brands in Films: Young Movie- } \\
\text { goers' Experiences and Interpreta- } \\
\text { tions }\end{array}$ & $\begin{array}{l}\text { DeLorme, Reid y Zim- } \\
\text { mer }\end{array}$ & 1994 & $\begin{array}{l}\text { Exposición a las marcas emplazadas } \\
\text { en un largometraje. }\end{array}$ \\
\hline $\begin{array}{l}\text { Effects of Brand Placements in } \\
\text { Feature Films }\end{array}$ & Karrh & 1994 & $\begin{array}{l}\text { Prominencia, repetición, integración } \\
\text { en la trama }\end{array}$ \\
\hline
\end{tabular}

Feature Films

Karrh en la trama

The Role of Thematic Congruence

Between a Mood-Inducing Event and an Advertised Product in Howard y Barry

1994 Congruencia temática

Determining the Effects of Mood and Brand Attitudes

Brand Recall for Product Placements in Motion Pictures: A

Memory-Based Perspective

Product Placements in the Top

Grossing Films of 1991

A Review And Investigation Of Into The Effectiveness Of Product

Placements In Films

The Impact On Children Brands

Of And Product Placements In

Films

Viewers' Recognition of Brands

Placed Within a Film

All Cues Are Not Created Equal:

Obtaining Attitude Persistent

Under Low-Involvement Condi-

tions

The Effects of Brand Placement

Type and a Disclaimer on

Memory for Brand Placements in

Movies.

Consumer's Perceptions of the

Ethics and Acceptability of Product Placement in Movies: Product

Category and Individual Differ-

ences

Product Placement in Movies. The

Effect of Prominence and Mode

on Audience Recall

The Influence of Product-

Placement Type and Exposure

Time on Product-Placement

Recognition
Gupta y Lord

Brennan, Dubas y Babin
Sabherwahl,
Pokrywcynski y Griffin

Sapolsky y Kinney

Gupta y Gould

Sengupta, Goodstein y

Baninger

Vollmers y Mizerski

Vollmers

Babin y Carder

1996

1994

994

Presentación del estímulo: visual y audiovisual.

Género de película, categoría de producto, uso de producto, tiempo de exposición, interacción con el producto y nivel de implicación con el producto.

Tipo de exposición (marca emplazada/emplazamiento sin marca).

995 Tipo del emplazamiento. da/emplazamiento sin marca).

Implicación en el procesamiento, tiempo de exposición y tipo de estímulo (central y periférico).

Ubicación del emplazamiento, presentación del estímulo (visual, auditivo y

Zimmer y DeLorme

1997 audiovisual), tono del emplazamiento (humor) y uso por parte de los personajes.

997

Categoría de producto y diferencias individuales.
Prominencia, presentación del estímulo (visual, auditivo y audiovisual).
Tipo de emplazamiento (creativo y en escena) y tiempo de exposición. 


\begin{tabular}{|c|c|c|c|}
\hline TÍTULO & AUTOR & $A \tilde{N} O$ & VARIABLES \\
\hline $\begin{array}{l}\text { A Study of Factors Affecting } \\
\text { Consumer Evaluations and } \\
\text { Memory of Product Placements in } \\
\text { Movies }\end{array}$ & D'Astous y Chartier & 2000 & $\begin{array}{l}\text { Prominencia, integración con la } \\
\text { escena, obviedad del emplazamiento y } \\
\text { presencia del actor principal. }\end{array}$ \\
\hline $\begin{array}{l}\text { I'll Have What She's Having: } \\
\text { Gauging the Impact of Product } \\
\text { Placements on Viewers }\end{array}$ & Law y Braun & 2000 & Prominencia \\
\hline $\begin{array}{l}\text { Placements As Mediators Of } \\
\text { Brand Salience Within A UK } \\
\text { Cinema Audience }\end{array}$ & Johnstone y Dodd & 2000 & Exposición previa al emplazamiento. \\
\hline $\begin{array}{l}\text { Brand Appearances In Contempo- } \\
\text { rary Cinema Films And Contribu- } \\
\text { tion To Global Marketing Of } \\
\text { Cigarettes }\end{array}$ & $\begin{array}{l}\text { Sargent, Tickle, Beach, } \\
\text { Dalton, Ahrens, Heather- } \\
\text { ton }\end{array}$ & 2001 & $\begin{array}{l}\text { Tipo de aparición de la marca (como } \\
\text { fondo o usada por algún actor). }\end{array}$ \\
\hline $\begin{array}{l}\text { Self-Congruency as a Cue in } \\
\text { Different Advertising-Processing } \\
\text { Contexts }\end{array}$ & Chang & 2002 & $\begin{array}{l}\text { Implicación con el producto, estado de } \\
\text { ánimo y auto-congruencia }\end{array}$ \\
\hline $\begin{array}{l}\text { Product Placement in Movies: A } \\
\text { Comparison of Chinese and } \\
\text { American Consumers' Attitudes }\end{array}$ & McKechnie y Zhou & 2003 & $\begin{array}{l}\text { Hábitos de los consumidores (asisten- } \\
\text { cia al cine), categoría de producto y } \\
\text { país. }\end{array}$ \\
\hline $\begin{array}{l}\text { "Exploring Children's Choice: } \\
\text { The Reminder Effect of Product } \\
\text { Placement", }\end{array}$ & Auty y Lewis & 2004 & $\begin{array}{l}\text { Exposición previa al emplazamiento, } \\
\text { tipo de exposición (marca emplaza- } \\
\text { da/emplazamiento sin marca). }\end{array}$ \\
\hline $\begin{array}{l}\text { Effectiveness of Product Place- } \\
\text { ments in Indian Films and its } \\
\text { effects on Brand Memory and }\end{array}$ & Panda & 2004 & Modalidad y conexiones con la trama. \\
\hline
\end{tabular}

Attitude with Special Reference to

Hindi Films

Tabla 2. Estudios sobre emplazamiento de producto en televisión.

\begin{tabular}{|c|c|c|c|}
\hline TÍTULO & AUTOR & $\boldsymbol{A} \tilde{N} \boldsymbol{O}$ & VARIABLES \\
\hline $\begin{array}{l}\text { Happy and Sad TV Programs: } \\
\text { How They Affect Reactions to } \\
\text { Commercials }\end{array}$ & Goldberg y Gorn & 1987 & $\begin{array}{l}\text { Estado de ánimo inducido por el } \\
\text { programa. }\end{array}$ \\
\hline $\begin{array}{l}\text { Consumer Reactions to Product } \\
\text { Placement Strategies in Television } \\
\text { Sponsorship }\end{array}$ & D'Astous y Séguin & 1999 & $\begin{array}{l}\text { Tipo de emplazamiento, imagen del } \\
\text { patrocinador, tipo del programa de } \\
\text { televisión y congruencia entre el } \\
\text { patrocinador y el programa. }\end{array}$ \\
\hline $\begin{array}{l}\text { "Verisimilitude or advertising? } \\
\text { Brand appearances on prime-time } \\
\text { television" }\end{array}$ & Avery y Ferraro & 2000 & $\begin{array}{l}\text { Tipo y tono del programa, modo de } \\
\text { presentación (verbal, auditiva y } \\
\text { audiovisual), prominencia y tipo de } \\
\text { implicación del personaje con el } \\
\text { emplazamiento (uso, portrayal, } \\
\text { mención o todos). }\end{array}$ \\
\hline $\begin{array}{l}\text { Brand appearances on prime-time } \\
\text { television }\end{array}$ & Ferraro y Avery & 2000 & $\begin{array}{l}\text { Modalidad (verbal, auditiva y audio- } \\
\text { visual) y conexión con la trama, } \\
\text { prominencia, número de referencias } \\
\text { a la marca en una escena, importan- } \\
\text { cia de la referencia, contexto (tono } \\
\text { del programa). }\end{array}$ \\
\hline
\end{tabular}




\begin{tabular}{|c|c|c|c|}
\hline TÍTULO & AUTOR & $\boldsymbol{A} \tilde{N} \boldsymbol{O}$ & VARIABLES \\
\hline $\begin{array}{l}\text { Investigating the Effectiveness of } \\
\text { Product Placements in Television } \\
\text { Shows: The Role of Modality and } \\
\text { Plot Connection Congruence on } \\
\text { Brand Memory and Attitude }\end{array}$ & Russell & 2002 & $\begin{array}{l}\text { Modalidad (verbal, auditiva y audio- } \\
\text { visual) y conexión con la trama. }\end{array}$ \\
\hline $\begin{array}{l}\text { Product placement: How Brands } \\
\text { Appear on Television }\end{array}$ & La Ferle y Edwards & 2006 & $\begin{array}{l}\text { Modalidad del emplazamiento, } \\
\text { tiempo de exposición, tipo de empla- } \\
\text { zamiento (anuncio, logo, texto), } \\
\text { prominencia, tipo de exposición } \\
\text { (completa, parcial), significado del } \\
\text { emplazamiento, saturación, plano, } \\
\text { redundancia, encuadre, contexto } \\
\text { (tono del programa), interacción con } \\
\text { el personaje, categoría de producto. }\end{array}$ \\
\hline $\begin{array}{l}\text { Come on Down: How Consumers } \\
\text { View Game Shows and the Prod- } \\
\text { ucts Placed in Them }\end{array}$ & Gould y Gupta & 2006 & $\begin{array}{l}\text { Tipo de exposición de marcas em- } \\
\text { plazadas en concursos televisivos. }\end{array}$ \\
\hline $\begin{array}{l}\text { Consumers, Characters, and Prod- } \\
\text { ucts: A Balance Model of Sitcom } \\
\text { Product Placement Effects }\end{array}$ & Russell y Stern & 2006 & $\begin{array}{l}\text { Valencia del emplazamiento con el } \\
\text { personaje y fuerza de la relación del } \\
\text { emplazamiento con el personaje. }\end{array}$ \\
\hline $\begin{array}{l}\text { Understanding Attitudes Toward } \\
\text { And Behaviours In Response To } \\
\text { Product Placement }\end{array}$ & De Gregorio y Sung & 2010 & $\begin{array}{l}\text { Características demográficas y } \\
\text { sociológicas de los espectadores } \\
\text { (tanto de cine como de televisión). }\end{array}$ \\
\hline
\end{tabular}

\section{Tabla 3. Estudios sobre emplazamiento de producto en videojuegos.}

\begin{tabular}{|c|c|c|c|}
\hline TÍTULO & AUTOR & $\boldsymbol{A} \tilde{N} \boldsymbol{O}$ & $V A R I A B L E S$ \\
\hline $\begin{array}{l}\text { Recall of Brand Placements in } \\
\text { Computer/Video Games }\end{array}$ & Nelson & 2002 & Tipo de marca (real/ficticia) \\
\hline $\begin{array}{l}\text { The Effect of Billboards within } \\
\text { the Gaming Environment }\end{array}$ & Chaney, Lin y Chaney & 2004 & $\begin{array}{l}\text { Experiencia de juego y categoría de } \\
\text { producto. }\end{array}$ \\
\hline $\begin{array}{l}\text { Advertainment or Adcreep? Game } \\
\text { Players' Attitudes toward Adver- } \\
\text { tising and Product Placements in } \\
\text { Computer Games }\end{array}$ & Nelson, Keum y Yaros & 2004 & $\begin{array}{l}\text { Valencia de las opiniones (positiva, } \\
\text { negativa o neutra). }\end{array}$ \\
\hline $\begin{array}{l}\text { Hispanic Attitudes toward Adver- } \\
\text { games: A Proposed Model of their } \\
\text { Antecedents }\end{array}$ & $\begin{array}{l}\text { Hernández, Chapa, } \\
\text { Minor, Maldonado y } \\
\text { Barranzuela }\end{array}$ & 2004 & $\begin{array}{l}\text { Congruencia, intrusión, tiempo de } \\
\text { exposición, entretenimiento, irritación } \\
\text { y género de videojuego. }\end{array}$ \\
\hline $\begin{array}{l}\text { Experiencing Interactive Advertis- } \\
\text { ing Beyond Rich Media: Impacts } \\
\text { of Ad Type and Presence on } \\
\text { Brand Effectiveness in 3D Gam- } \\
\text { ing Immersive Virtual Environ- } \\
\text { ments }\end{array}$ & Grigorovici y Constantin & 2004 & $\begin{array}{l}\text { Tipo de emplazamiento (on-set, } \\
\text { creativo) e implicación con el video- } \\
\text { juego (alta, baja). }\end{array}$ \\
\hline $\begin{array}{l}\text { Cashing in on crashes via brand } \\
\text { placement in computer games: } \\
\text { The effects of experience and } \\
\text { flow on memory }\end{array}$ & Schneider y Cornwell & 2005 & $\begin{array}{l}\text { Prominencia espacial del emplaza- } \\
\text { miento (alta, baja), estado de flujo y } \\
\text { experiencia de juego. }\end{array}$ \\
\hline $\begin{array}{l}\text { The Effect of Involvement on Ad } \\
\text { Judgment in a Video Game Envi- } \\
\text { ronment: The Mediating Role of }\end{array}$ & Nicovich & 2005 & Implicación y presencia. \\
\hline
\end{tabular}




\begin{tabular}{|c|c|c|c|}
\hline TÍTULO & AUTOR & $A \tilde{N} O$ & $V A R I A B L E S$ \\
\hline $\begin{array}{l}\text { Examining The Influence Of } \\
\text { Telepresence On Spectator And } \\
\text { Player Processing Of Real and } \\
\text { Fictitious Brands in a Computer } \\
\text { Game }\end{array}$ & Nelson, Yaros y Keum & 2006 & $\begin{array}{l}\text { Contexto del medio (juga- } \\
\text { do/visionado), tipo de marca } \\
\text { (real/ficticia) y telepresencia. }\end{array}$ \\
\hline $\begin{array}{l}\text { Receptiveness of Gamers to } \\
\text { Embedded Brand Messages in } \\
\text { Advergames: Attitudes towards } \\
\text { Product Placement }\end{array}$ & Winkler y Buckner & 2006 & $\begin{array}{l}\text { Tipo de advergame (asociativo, ilus- } \\
\text { trativo y demostrativo) y género de de } \\
\text { videojuego. }\end{array}$ \\
\hline $\begin{array}{l}\text { Real Brands in Imaginary Worlds: } \\
\text { Investigating Players' Experiences } \\
\text { of Brand Placement in Digital } \\
\text { Games }\end{array}$ & Molesworth & 2006 & Experiencias de juego. \\
\hline $\begin{array}{l}\text { The Effectiveness of 'in-game' } \\
\text { advertising: Comparing Collage } \\
\text { Students' Explicit and Implicit } \\
\text { Memory for Brand Names }\end{array}$ & $\begin{array}{l}\text { Yang, Roskos-Ewoldsen, } \\
\text { Dinu y Arpan }\end{array}$ & 2006 & Género de videojuego. \\
\hline $\begin{array}{l}\text { Effects of Product Placement in } \\
\text { On-Line Games on Brand } \\
\text { Memory: A Perspective of the } \\
\text { Limited Capacity Model of Atten- } \\
\text { tion }\end{array}$ & Lee y Faber & 2007 & $\begin{array}{l}\text { Proximidad (focal/periférica), con- } \\
\text { gruencia, implicación con el videojue- } \\
\text { go (alta/moderada) y experiencias de } \\
\text { juego. }\end{array}$ \\
\hline $\begin{array}{l}\text { The Effects of Playing an Adver- } \\
\text { game on Young Children's Per- } \\
\text { ceptions, Preferences, and Re- } \\
\text { quests }\end{array}$ & Mallinckrodt y Mizerski & 2007 & $\begin{array}{l}\text { Tipo de exposición (marca emplaza- } \\
\text { da/emplazamiento sin marca). }\end{array}$ \\
\hline $\begin{array}{l}\text { The Effectiveness of Product } \\
\text { Placement in Video Games }\end{array}$ & Glass & 2007 & $\begin{array}{l}\text { Marcas emplazadas/marcas no empla- } \\
\text { zadas }\end{array}$ \\
\hline $\begin{array}{l}\text { Enjoyment of Advergames and } \\
\text { Brand Attitudes: The Impact of } \\
\text { Thematic Relevance }\end{array}$ & $\begin{array}{l}\text { Wise, Bolls, Kim, Ven- } \\
\text { kataraman, y Meyer }\end{array}$ & 2008 & $\begin{array}{l}\text { Conexión temática (congruencia) y } \\
\text { género de videojuego. }\end{array}$ \\
\hline $\begin{array}{l}\text { Communicating brands playfully: } \\
\text { Effects of in-game advertising for } \\
\text { familiar and unfamiliar brands }\end{array}$ & Mau, Silberer y Constien & 2008 & Familiaridad con la marca \\
\hline $\begin{array}{l}\text { Advergames: The Impact of } \\
\text { Brand Prominence and Game } \\
\text { Repetition on Brand Responses }\end{array}$ & $\begin{array}{l}\text { Cauberghe y De Pelsma- } \\
\text { cker }\end{array}$ & 2010 & Prominencia y tiempo de juego \\
\hline $\begin{array}{l}\text { In-Game Advertising Effects: } \\
\text { Examining Player Perceptions Of } \\
\text { Advertising Schema Congruity In } \\
\text { A Massively Multiplayer Online } \\
\text { Role-Playing Game }\end{array}$ & Lewis y Porter & 2010 & $\begin{array}{l}\text { Congruencia del emplazamiento y } \\
\text { experiencia de juego. }\end{array}$ \\
\hline
\end{tabular}

\section{Análisis de la muestra}

La muestra fue sometida a un análisis de contenido con el fin de identificar las variables independientes presentes en los trabajos empíricos analizados. Otras variables como el medio en el que se producía el emplazamiento de producto analizado en el trabajo (cine, televisión o videojuegos), el título del trabajo, el autor o autores y el año también fueron registradas. Fruto de este análisis se detectó que las variables relacionadas con la localización, ubicación, prominencia, tipo de empla- 
zamiento o estímulo (en escena o creativo) y proximidad, estaban presentes en 21 estudios; la congruencia, integración o conexión con la trama estaban presentes en 11 estudios; las variables relacionadas con la modalidad del emplazamiento y la presentación del estímulo (verbal, visual y audiovisual), estaban presentes en 9 estudios; las variables relacionadas con el género de película, el tipo de programa televisivo y el género de videojuego en el que se produce el emplazamiento estaban presentes en 9 estudios; la interacción o uso por parte de los personajes estaba presente en 8 estudios; la implicación (con el medio, mensaje o producto) estaba presente en 6 estudios; la categoría de producto del emplazamiento estaba presente en 6 estudios; la duración o tiempo de exposición del emplazamiento estaba presente en 6 estudios; la experiencia de juego del emplazamiento estaba presente en 5 estudios; y el tipo de marca del emplazamiento estaba presente en 2 estudios. Otra serie de variables aparecían únicamente analizadas en un solo artículo, tales como: la fuerza de la relación del emplazamiento con el personaje, la valencia del emplazamiento con el personaje, los hábitos de los consumidores (asistencia al cine), el estado de ánimo, el tono del emplazamiento, la saturación (presencia de otras marcas de forma simultánea en el emplazamiento), el tipo de plano o encuadre, etc. El análisis de estas variables independientes utilizadas en los estudios empíricos analizados permitió identificar y conceptualizar una serie de dimensiones principales (con sus correspondientes variables) que fueron utilizadas para el desarrollo de la herramienta.

\section{Desarrollo de la herramienta}

Con el fin de desarrollar una herramienta lo más operativa posible se discriminaron las variables que podían ser agrupadas en categorías que presentaran un carácter dicotómico (¿emplazamiento visual o verbal? ¿emplazamiento en escena o creativo?) de aquellas que podían ser comunes a más de una categoría (por ejemplo, la valencia del emplazamiento). De esta forma se realizó un agrupamiento en lo que denominados variables dicotómicas y variables genéricas. Esta clasificación dicotómica ya fue utilizada por Spiegelman, Terwilliger y Fearing (1953) en un análisis de contenido que realizaron sobre el lugar en el que ocurrían las aventuras de los cómics. Para ello utilizaron preguntas dicotómicas del tipo: A) ¿ocurren en la Tierra o en el espacio? B) si es en la Tierra, ¿en los EE.UU o en el extranjero? C) ¿en el entorno rural o urbano? La estructura ramificada así obtenida permite obtener un organigrama que va acumulando diversas dimensiones y variables que permite obtener una serie de categorías de emplazamiento de producto de complejidad creciente. En esta primera fase también se eliminaron todas aquellas variables individuales (características demográficas de los consumidores, implicación con la categoría de producto, estado de ánimo del consumidor, etc.) que requieren de otro tipo de herramientas para ser capturadas (por ejemplo, cuestionarios respondidos 
por los propios consumidores) y que, por lo tanto, no pueden ser capturadas mediante un análisis de contenido. Por ejemplo, las características personales de un espectador determinarán si juzga como congruente o incongruente un emplazamiento de una marca o producto en un contexto determinado (al igual que la familiaridad que pueda tener con esa marca o el apego parasocial que haya podido desarrollar hacia un personaje de una comedia de situación) pero no pueden ser recogidas mediante un análisis de contenido.

\subsection{Variables dicotómicas}

A partir del análisis de la literatura académica revisada se conceptualizaron las siguientes dimensiones que contienen las diferentes variables identificadas: modalidad, grado de integración, proximidad, dimensionalidad, alocución, estado y diégesis narrativa. Estas variables dicotómicas se recogen en la tabla 4.

Tabla 4. Variables Dicotómicas.

\begin{tabular}{|c|l|}
\hline DIMENSIÓN & \multicolumn{1}{c|}{ VARIABLE } \\
\hline Modalidad & Emplazamiento visual o emplazamiento auditivo \\
\cline { 2 - 2 } & Emplazamiento verbal o emplazamiento sonoro \\
\hline Grado de integración & $\begin{array}{l}\text { Emplazamiento que forma parte del escenario o emplazamiento } \\
\text { que forma parte de la trama/acción }\end{array}$ \\
\hline Proximidad & $\begin{array}{l}\text { Emplazamiento situado como fondo (periférico) o emplaza- } \\
\text { miento en un plano próximo (focal) }\end{array}$ \\
\hline Dimensionalidad & $\begin{array}{l}\text { Emplazamiento de un objeto o emplazamiento de una imagen } \\
\text { sobre impresionada }\end{array}$ \\
\hline Alocución & Mención verbal por parte de un personaje o voz en off \\
\hline Estado & Emplazamiento estático o emplazamiento animado \\
\hline Diégesis narrativa & $\begin{array}{l}\text { Forma parte de la diégesis narrativa o no forma parte de la } \\
\text { diégesis narrativa }\end{array}$ \\
\hline
\end{tabular}

\subsubsection{Modalidad}

La modalidad del emplazamiento viene determinada por el sistema de codificación del lenguaje. Basándose en el sistema de codificación dual de Paivio (1983, 1986), esta modalidad puede ser verbal (procesamiento del lenguaje) o no verbal (procesamiento de las imágenes). Para esta dimensión se han conceptualizado dos variables: emplazamiento visual y emplazamiento auditivo. Se utiliza el término auditivo (y no verbal) ya que este tipo de emplazamientos pueden ser tanto verbales como sonoros (por ejemplo, el sonido registrado de Intel Inside o el sonido corporativo que identifica un tono de llamada con una determinada marca de teléfonos móviles). 


\subsubsection{Grado de integración}

Esta dimensión recoge el grado de integración de la marca o producto en el contenido audiovisual. Así un emplazamiento está poco integrado cuando únicamente forma parte del decorado o atrezzo del entorno en el que se desarrolla la película, serie o videojuego. Por el contrario, se considera que un emplazamiento está muy integrado cuando forma parte de la trama o acción que se desarrolla en dicho contenido audiovisual ${ }^{37}$. En este sentido, la dimensión comprende dos variables: emplazamiento en pantalla y emplazamiento en guión.

\subsubsection{Proximidad}

El concepto de proximidad hace referencia a la ubicación de la marca emplaza$\mathrm{da}^{38}$. Este concepto está relacionado con el concepto de prominencia ${ }^{39}$ y determina el grado en el que un emplazamiento tiene una mayor capacidad para captar la atención del consumidor. Así, en la literatura se ha diferenciado entre emplazamientos en escena (o focales) y emplazamientos creativos (o periféricos) en función de esta ubicación ${ }^{40}$. Un emplazamiento en escena se correspondería, por ejemplo, con un producto emplazado bien visible en la mesa en la que está sentado el actor de una película mientras que un emplazamiento creativo podría estar emplazado en una estantería al fondo de la cocina. En el medio cinematográfico existe una evidente correlación entre esta proximidad de la marca emplazada y el tipo de plano utilizado para mostrarla al espectador. Así, un emplazamiento en escena suele ser mostrado mediante un primer plano mientras que los emplazamientos creativos aparecen generalmente en los planos generales. En esta dimensión se han considerado dos variables: emplazamiento focal y emplazamiento periférico.

\subsubsection{Dimensionalidad}

Esta dimensión hace referencia a la naturaleza bidimensional o tridimensional de la marca u objeto emplazados. Los emplazamientos pueden ser por tanto objetos tridimensionales (por ejemplo, un coche, un reloj o unas zapatillas) u objetos bidimensionales sobre impresionados o estampados sobre diversas superficies (por ejemplo, sobre una taza, una camiseta o el tapete de una mesa de billar). Esta di-

37 Russell, C. A. (2002): «Investigating the Effectiveness of Product Placements in Television Shows: The Role of Modality and Plot Connection Congruence on Brand Memory and Attitude», Journal of Consumer Research, 29 (3), 306-318.

38 LeE, M.; FABER, R.J. (2007): «Effects of Product Placement in On-Line Games on Brand Memory», Journal of Advertising, 36 (4), 75-90

39 GuptA, P. B.; LoRD, K. R. (1998): «Product Placement in Movies. The Effect of Prominence and Mode on Audience Recall», Journal of Current Issues and Research in Advertising, 20 (1), 47-59. SCHNEIDER, L. P.; CORNwELL, B. (2005): «Cashing in on crashes via brand placement in computer games: The effects of experience and flow on memory», International Journal of Advertising, 24, (3), 321-343.

40 Brennan, I.; Dubas K. M.; BABIN L. A. (1999): «The Influence of Product-Placement Type and Exposure Time on Product-Placement Recognition» International Journal of Advertising, 18 (3), 323-337. 
mensión comprende dos variables: emplazamiento bidimensional y emplazamiento tridimensional.

\subsubsection{Alocución}

Esta dimensión diferencia entre dos tipos de emplazamientos verbales: aquellos pronunciados por los personajes (por ejemplo, cuando el actor protagonista pide en un bar una determinada marca de cerveza) o las voces en off (por ejemplo, una cuña radiofónica que se oye de fondo mientras se desarrolla la escena). Esta dimensión resulta importante para diferenciar el tipo de respaldo verbal que puede producirse en un contenido audiovisual por parte de un actor que tenga una influencia sobre el espectador ${ }^{41}$ de otro tipo de emplazamientos verbales más neutros. Esta dimensión comprende dos variables: emplazamiento alucoción y emplazamiento voz en off.

Finalmente se introdujeron dos dimensiones (estado y diégesis narrativa) que aunque no se encontraron presentes en ninguno de los trabajos analizados se consideró oportuno añadir por sus implicaciones a la hora de tipificar determinados emplazamientos.

\subsubsection{Estado}

Esta dimensión recoge una característica cada vez más presente en los emplazamientos de producto: la animación del estímulo presentado. Se sabe que cualquier cambio en un estímulo presentado a un individuo (por ejemplo, el parpadeo de un letrero luminoso o el movimiento de una valla publicitaria) tiene importantes consecuencias sobre el procesamiento de la información del estímulo realizado por el consumidor. De hecho, la animación de un objeto emplazado en un fondo estático ha sido identificada como una característica distintiva de los anuncios ${ }^{42}$. Esta dimensión comprende dos variables: emplazamiento estático y emplazamiento animado.

41 Russell, C. A.; STERN, B. B. (2006): «Consumers, Characters, and Products: A Balance Model of Sitcom Product Placement Effects», Journal of Advertising, 35 (1), 7-21.

42 Beattie, A. E.; Mitchell A. A (1985): «The Relationship between Advertising Recall and Persuasion: An Experimental Investigation», en AlwitT, L. F.; Mitchell, A. A (eds), Psychological Processes and Advertising Effects: Theory, Research, and Application, Hillsdale (Nueva Jersey), Erlbaum, 129155. TAYloR, S. E.; ThOMPSON, S. C. (1982): «Stalking the Elusive Vividness Effect», Psychological Review, 89 (2), 155-81. GATI, I.; TVERSKY, A. (1987): «Recall of Common and Distinctive Features of Verbal and Pictorial Stimuli», Memory \& Cognition, 15 (2), 97-100. NAIRNE, J. S.; NEATH, I., SERRA, M.; BYUN, E. (1997): «Positional Distinctiveness and Ratio Rule in Free Recall», Journal of Memory \& Language, 37 (3), 155-166. 


\subsubsection{Diégesis narrativa}

Esta categoría identifica si un emplazamiento auditivo forma parte del mundo ficcional audiovisual o no. Por ejemplo, una canción que está escuchando uno de los protagonistas de una película en la radio de su coche forma parte de la diégesis narrativa mientras que la banda sonora que suena cuando los dos protagonistas se besan (pero que ellos no oyen) no forma parte de esta diégesis narrativa. Esta dimensión comprende dos variables: emplazamiento diegético y emplazamiento no diegético.

\subsection{Variables genéricas}

Junto a las variables dicotómicas, las variables genéricas permiten obtener datos sobre el propio contenido audiovisual analizado (por ejemplo, el género de película, programa de televisión o videojuego) y la marca o producto emplazados a la vez que capturan diversas variables que aportan valor al emplazamiento (por ejemplo, la valencia del emplazamiento, su relevancia en la trama/acción, el tiempo de exposición del emplazamiento, etc.). Estas variables genéricas fueron agrupadas en las siguientes categorías: datos generales, características de la marca emplazada y variables de valor.

\subsubsection{Datos generales}

Junto a los datos considerados generales (por ejemplo los relativos al propio documento audiovisual que forme parte de la muestra de análisis) se incorporaron otras variables comunes a las herramientas de análisis de contenido que permiten obtener un control sobre el proceso (por ejemplo, la fecha de análisis o el evaluador). Así pues los datos generales contemplan las siguientes variables: título del documento audiovisual analizado, año de producción, género, fecha de análisis y evaluador.

\subsubsection{Datos identificadores de la marca emplazada}

Entre las características de la marca se identificaron las siguientes variables: nombre de la marca, categoría de producto y tipo de marca. En la categoría tipo de marca se incorporaron como variables de análisis a la distinción clásica entre real y ficticia ${ }^{43}$ las variables de marca enmascarada y marca mejorada. Estas últimas variables han sido identificadas recientemente ${ }^{44}$ como tipologías emergentes en el emplazamiento de producto. Las marcas enmascaradas hacen referencia a «marcas ficticias cuyos elementos de diseño (elementos gráficos, tipográficos, etc.) y de uso (correspondiente a la categoría de producto de la marca real) permiten una

43 Nelson, M. R. (2002): «Recall of Brand Placements in Computer/Video Games», Journal of Advertising Research, 42 (2), 80-92.

44 MARTí, J.; ALDÁs, J.; CURRÁs, R.; SÁNCHEZ, I. (2010): «El emplazamiento de producto: conceptualización, nuevos formatos y efectos sobre el consumidor», Teoría y Praxis, 8, 113-136. 
asociación inmediata entre las marcas ficticias y las marcas reales a las que representan ${ }^{45}$. Por otra parte, las marcas mejoradas han sido conceptualizadas como «marcas o productos reales cuyas características y propiedades han sido modificadas y mejoradas virtualmente con el objetivo de que el espectador atribuya estas nuevas características y beneficios a la marca o producto "real" $\gg$.

\subsection{Variables de valor}

Por lo que respecta a las variables de valor se recogen todas las categorías que ayudan a caracterizar el nivel de emplazamiento identificado mediante las variables dicotómicas. Entre estas variables de valor se encuentran: bimodalidad, codificación, relevancia, interacción, valencia, tiempo de exposición, redundancia, saturación, rango del personaje y personalidad del personaje.

\subsubsection{Bimodalidad}

Aunque tradicionalmente los emplazamientos de producto han utilizado una de las dos modalidades de forma excluyente (o bien se veía la maca/producto o bien era nombrada) cada vez es más frecuente detectar emplazamientos bimodales que utilizan de forma simultánea ambas modalidades. Por ejemplo, en una secuencia de la película Ultimátum a la Tierra (Scott Derrickson, 2008) la protagonista exclama sorprendida "¿McDonald's?" mientras su coche se detiene frente a uno de estos establecimientos y el logotipo de la marca se ve reflejado en el parabrisas del vehículo. Desde esta perspectiva, esta variable identifica si un emplazamiento visual (auditivo) es reforzado simultáneamente con un emplazamiento auditivo (visual), dando lugar a un emplazamiento bimodal y, por tanto, audiovisual.

\subsubsection{Codificación}

Esta variable tiene en cuenta el modo en el que ha sido codificada la información visual presentada al consumidor. Se han considerado tres variables: textual, icónica o gráfica. Por codificación textual se entenderá toda información semántica (palabras o cualquier otro tipo de texto representado gráficamente); por codificación icónica se entenderá toda información presentada mediante imágenes (logotipos, colores corporativos, etc.); y por codificación gráfica se entenderá toda información presentada mediante un uso conjunto de las anteriores (por ejemplo, un anuncio que presenta tanto imágenes como palabras).

\subsubsection{Relevancia}

Esta variable identifica si el emplazamiento tiene una alta/media/baja relevancia con respecto a la trama/acción del contenido audiovisual. Por ejemplo, si el emplaza-

\footnotetext{
45 Ibídem, 121.

46 Ibidem, 124.
} 
miento de una marca o producto aporta una pista al personaje o su uso resulta determinante en una acción (en el caso de los videojuegos o los relatos interactivos).

\subsubsection{Interacción}

Esta variable determina si se produce algún tipo de interacción entre el agente (personaje de un relato de ficción, presentador de un espacio televisivo, jugador de un videojuego, etc.) y el producto o la marca emplazada. Por ejemplo, si un actor bebe de una lata de cerveza cuya marca es reconocible o si un videojugador puede conducir un coche de una marca determinada. Esta dimensión resulta importante ya que se ha detectado que, en el contexto de los videojuegos, la interacción con las marcas emplazadas influye en la memoria de los videojugadores ${ }^{47}$. Esta dimensión comprende dos variables: emplazamiento interactivo y emplazamiento no interactivo. Siguiendo la clasificación de Sapolsky y Kinney (1994) se determinaron los siguientes grados para los emplazamientos interactivos: el producto se sujeta/toca, se $u s a$, se consume.

\subsubsection{Valencia}

Esta variable evalúa si el emplazamiento tiene una carga positiva (por ejemplo, un juicio positivo por parte de un personaje), neutra (por ejemplo, la marca simplemente está ahí) o negativa (por ejemplo, se efectúa una crítica hacia la marca o producto emplazado).

\subsubsection{Tiempo de exposición}

Esta variable mide en segundos el tiempo de exposición de la marca en la pantalla.

\subsubsection{Redundancia}

Mediante esta variable se mide el número de apariciones de la marca en el contenido audiovisual identificando el orden de aparición de la marca: si es la $1^{\mathrm{a}}$ vez que aparece, la $2^{\mathrm{a}}$ vez, etc.

\subsubsection{Saturación}

Esta variable mide el número de otras marcas presentes simultáneamente durante el momento del emplazamiento.

\subsubsection{Rango del personaje}

Esta variable identifica el papel del personaje que se relaciona con el emplazamiento. Siguiendo la propuesta de La Ferle y Edwards (2006) se consideran las siguientes categorías: protagonista, secundario, extra, celebridad y experto. Con el

47 SCHNEIDER, L. P.; CORNwELl, B. (2005): «Cashing in on crashes via brand placement in computer games: The effects of experience and flow on memory», International Journal of Advertising, 24, (3), 321343. 
fin de ampliar el rango de los personajes a contenidos audiovisuales de no ficción como los telediarios o los reality shows, etc., se ha considerado también incluir la categoría presentador televisivo.

\subsubsection{Personalidad del personaje}

Esta variable identifica la personalidad del personaje relacionado con el emplazamiento. Siguiendo la clasificación de Sapolsky y Kinney (1994) se determinaron tres variables: bueno, malo y neutral.

\section{Herramienta para el análisis de contenido del emplazamiento de producto en contenidos audiovisuales}

El análisis de las diferentes variables utilizadas en la literatura académica para estudiar el emplazamiento de producto permitió identificar una serie de dimensiones (y sus correspondientes variables) que, a su vez, permitieron una sistematización en la identificación de una serie de niveles de emplazamiento de producto en contenidos audiovisuales (la herramienta completa se recoge en la tabla 5). Puesto que el uso de variables dicotómicas permite ir agregando una serie de características a la muestra (¿emplazamiento visual o auditivo? ¿forma parte de la diégesis narrativa o no forma parte de la diégesis narrativa? ¿forma parte del escenario o forma parte de la trama/acción? etc.) también permite identificar diferentes categorías de emplazamiento con una creciente complejidad que abarcan desde un emplazamiento más sencillo (por ejemplo, un emplazamiento auditivo que forma parte de la banda sonora) hasta un emplazamiento más elaborado (por ejemplo, un emplazamiento visual que es utilizado por un personaje). Se espera que esta creciente complejidad esté asimismo correlacionada con los diferentes efectos (cognitivos, afectivos y conativos) que pueden generar los emplazamientos de producto en los consumidores $^{48}$. Por ejemplo, se espera que un emplazamiento auditivo que forma parte de la banda sonora genere una menor respuesta cognitiva (por ejemplo, manifestadas mediante el recuerdo del emplazamiento por parte del consumidor) que un emplazamiento visual que es utilizado por un personaje (por ejemplo, una cerveza de una determinada marca que es consumida por el protagonista principal ante el espectador). Del mismo modo, se espera que este emplazamiento visual que es utilizado por un personaje genere mayores respuestas afectivas que un emplazamiento visual de un objeto en un plano próximo (pero con el que no media ninguna interacción positiva por parte del personaje). Estos diferentes niveles identificados presentan las siguientes características:

Balasubramanian, S. K.; Karrh, J. A.; Patwardhan, H. (2006): «Audience Response to Product Placements: An Integrative Framework and Future Research Agenda», Journal of Advertising, 35 (3), $115-141$. 
- EMPLAZAMIENTO DE NIVEL 1: emplazamiento auditivo que forma parte de la banda sonora (por ejemplo, una canción de un grupo musical).

- EMPLAZAMIENTO DE NIVEL 2: emplazamiento sonoro que forma parte de la diégesis narrativa (por ejemplo, el sonido corporativo de los procesadores Intel Inside escuchado cuando un protagonista enciende un ordenador en una escena o el sonido de un móvil que identifica una determinada marca comercial).

- EMPLAZAMIENTO NIVEL 3: emplazamiento verbal de un narrador.

- EMPLAZAMIENTO NIVEL 4: emplazamiento verbal de un personaje.

- EMPLAZAMIENTO NIVEL 5: emplazamiento visual estático que forma parte del escenario como fondo (por ejemplo, una valla publicitaria).

- EMPLAZAMIENTO NIVEL 6: emplazamiento visual animado que forma parte del escenario como fondo (por ejemplo, un spot proyectado en una videowall o un letrero luminoso).

- EMPLAZAMIENTO NIVEL 7: emplazamiento visual de un objeto en un plano próximo (por ejemplo, un coche o una lata de refresco).

- EMPLAZAMIENTO NIVEL 8: emplazamiento visual de una imagen sobre impresionada en un plano próximo (por ejemplo, un logotipo en una camiseta o en una mesa de billar).

Tabla 5. Ficha propuesta para el análisis de contenido del emplazamiento de producto en contenidos audiovisuales.

\begin{tabular}{|l|l|}
\hline \multicolumn{1}{|c|}{ A. DATOS GENERALES } & \\
\hline Título del documento audiovisual analizado & \\
\hline Año de producción & \\
\hline Género & \\
\hline Fecha de análisis & \\
\hline Evaluador & \\
\hline
\end{tabular}

\begin{tabular}{|l|l|}
\hline \multicolumn{2}{|c|}{ B. DATOS IDENTIFICADORES DE LA MARCA EMPLAZADA } \\
\hline Nombre de la marca & \\
\hline Categoría de producto & \\
\hline Tipo de marca & Real, ficticia, enmascarada, mejorada \\
\hline
\end{tabular}

\begin{tabular}{|l|l|}
\hline \multicolumn{1}{|c|}{ C. VARIABLES DE VALOR } \\
\hline Bimodalidad & Uso simultáneo de emplazamientos visuales y auditivos \\
\hline Codificación & Textual, icónica, gráfica \\
\hline Relevancia & Alta, media, baja \\
\hline Interacción & $\begin{array}{l}\text { Emplazamiento interactivo (la marca/producto se suje- } \\
\text { ta/toca/usa/consume) y emplazamiento no interactivo }\end{array}$ \\
\hline Valencia & Positiva, neutra, negativa \\
\hline Tiempo de exposición & Número de segundos que dura el emplazamiento \\
\hline Redundancia & Número de emplazamiento de la marca $\left(1^{\circ}, 2^{\circ} \ldots\right)$ \\
\hline
\end{tabular}




\begin{tabular}{|l|l|}
\hline \multicolumn{2}{|c|}{ C. VARIABLES DE VALOR } \\
\hline Saturación & $\begin{array}{l}\text { Número de marcas presentes simultáneamente durante el } \\
\text { momento del emplazamiento }\end{array}$ \\
\hline Rango del personaje & $\begin{array}{l}\text { Protagonista, secundario, extra, celebridad, experto y presen- } \\
\text { tador televisivo. }\end{array}$ \\
\hline Personalidad del personaje & Bueno, malo, neutral \\
\hline
\end{tabular}

\section{VARIABLES DICOTÓMICAS}

1. ¿Se trata de un emplazamiento visual (p.9) o auditivo (p.2)?

2. ¿Forma parte de la diégesis narrativa (p.4) o no (p.3)?

3. EMPLAZAMIENTO NIVEL 1: emplazamiento auditivo que forma parte de la banda sonora (por ejemplo, una canción de un grupo musical).

4. ¿Se trata de un emplazamiento verbal (p.6) o sonoro (p.5)?

5. EMPLAZAMIENTO NIVEL 2: emplazamiento sonoro que forma parte de la diégesis narrativa (por ejemplo, el sonido corporativo de los procesadores Intel Inside escuchado cuando un protagonista enciende un ordenador en una escena o el sonido de un móvil que identifica una determinada marca comercial).

6. ¿Se trata de una mención verbal por parte de un personaje (p.8) o una voz en off de un narrador que no es un personaje (p.7)?

7. EMPLAZAMIENTO NIVEL 3: emplazamiento verbal de un narrador.

8. EMPLAZAMIENTO NIVEL 4: emplazamiento verbal de un personaje.

9. ¿Se trata de un emplazamiento que forma parte del escenario (p.10) o de la trama/acción (p.17)?

10. ¿Se trata de un emplazamiento situado como fondo (p.11) o en un plano próximo (p.14)?

11. ¿Se trata de un emplazamiento estático (p.12) o animado (p.13)?

12. EMPLAZAMIENTO NIVEL 5: emplazamiento visual estático que forma parte del escenario como fondo (por ejemplo, una valla publicitaria).

13. EMPLAZAMIENTO NIVEL 6: emplazamiento visual animado que forma parte del escenario como fondo (por ejemplo, un spot proyectado en una videowall o un letrero luminoso).

14. ¿Se trata de un objeto (p.15) o de una imagen sobre impresionada (p.16)?

15. EMPLAZAMIENTO NIVEL 7: emplazamiento visual de un objeto en un plano próximo (por ejemplo, un coche o una lata de refresco).

16. EMPLAZAMIENTO NIVEL 8: emplazamiento visual de una imagen sobre impresionada en un plano próximo (por ejemplo, un logotipo en una camiseta o en una mesa de billar).

\section{Conclusiones}

Este trabajo presenta por primera vez una herramienta para el análisis de contenido de emplazamientos de marca/producto en una amplia variedad de contenidos audiovisuales. Una herramienta lo suficientemente versátil y sencilla de utilizar que facilite la tarea del investigador en un área de estudio emergente como es el emplazamiento producto. Mediante el análisis y la sistematización de las variables utilizadas en la literatura académica para estudiar el emplazamiento de producto se ha obtenido una herramienta que captura las principales dimensiones (modalidad, grado de integración, proximidad, dimensionalidad, alocución, estado y diégesis 
narrativa) y variables (emplazamiento visual, emplazamiento auditivo, emplazamiento en pantalla, emplazamiento tridimensional, etc.) que permiten asimismo tipificar una serie de categorías (concretadas en 8 niveles de emplazamiento) que presentan una creciente complejidad y riqueza en el tipo de emplazamiento. Esta creciente complejidad se espera que esté asimismo correlacionada con los diferentes efectos (cognitivos, afectivos y conativos) que pueden generar los emplazamientos de producto en los consumidores. La herramienta está compuesta por cuatro módulos de análisis (datos generales sobre el análisis a realizar, datos identificadores de la marca emplazada, variables de valor y variables dicotómicas) que permiten capturar los datos generales y las diferentes dimensiones y variables de los emplazamientos de producto en un amplio abanico de contenidos audiovisuales (tanto de ficción como de no ficción y en diferentes medios de difusión). Mediante esta herramienta se pretende facilitar la labor de los académicos que estudien los emplazamientos de producto en contenidos audiovisuales y conseguir una mejor, y más fiable, comparación entre los resultados de aquellos estudios que utilicen la herramienta.

\section{Limitaciones y futuras líneas de investigación}

Aunque la herramienta ha sido diseñada para poder ser aplicada a un amplio grupo de contenidos audiovisuales, las diferencias existentes entre los diferentes medios y géneros pueden dificultar su aplicación en algunas situaciones concretas. Por ejemplo, algunos géneros de videojuegos (como los mundos persistentes online) pueden presentar alguna dificultad a la hora de identificar los emplazamientos en función de la dimensión proximidad. Puesto que en este género de videojuego el jugador puede moverse libremente por el entorno del videojuego en ocasiones un emplazamiento que se presenta inicialmente como un emplazamiento periférico (es decir, situado al fondo de la escena) puede transformarse en un emplazamiento focal (es decir, en un emplazamiento situado próximo al jugador) como consecuencia de la acción de jugador. En este caso no habrá que olvidar que el tipo de emplazamiento vendrá determinado como consecuencia de la experiencia de juego de cada jugador (es decir, puede que un jugador haya decidido acercarse a una valla publicitaria que se encuentra al fondo, siendo de esta manera expuesto a un emplazamiento focal pero puede que otro jugador no lo haga, siendo por tanto expuesto a un emplazamiento periférico). Las implicaciones para el investigador en este caso le obligan a ser consciente de que la aplicación exitosa de esta herramienta debe realizarse pues sobre la base de una monitorización de la experiencia de juego de cada jugador. Por otra parte, el factor interactividad (ya sea en videojuegos, en la web, telefonía móvil o televisión, etc.) plantea una situación similar a la anterior. Es decir, aunque la naturaleza de un emplazamiento sea interactiva, el emplazamiento deberá ser tipificado en función de la actividad de cada usuario. Este trabajo 
exploratorio constituye un primer intento teórico por desarrollar un instrumento eficaz de análisis del emplazamiento de producto en un amplio abanico de contenidos audiovisuales. Es por ello que se requerirán nuevas reflexiones y análisis críticos de la misma. Asimismo resultará necesario realizar una validación empírica de la herramienta que determine su fiabilidad y validez como instrumento de análisis del emplazamiento de producto en el marco de los contenidos audiovisuales. Esta validación empírica deberá realizarse para los diferentes contenidos audiovisuales a los que se piensa que es aplicable de manera que pueda detectarse la necesidad de futuros ajustes o revisiones que hagan más eficaz este instrumento de análisis.

Por otra parte, el emplazamiento de producto es una herramienta de las comunicaciones de marketing en pleno desarrollo. Junto a los emplazamientos clásicos en medios audiovisuales como el cine y la televisión, los emplazamientos en otros tipos de medios como los impresos empiezan a ser cada vez más abundantes. Este hecho se pone especialmente de manifiesto en los bestsellers de ficción. Baste como ejemplo que en la novela de Dan Brown El símbolo perdido (Planeta Internacional, 2009) se nombran hasta 31 marcas que incluyen desde los motores Pratt \& Whitney hasta Volvo, Mercedes, Hummer, iPhone o Blackberry. En este sentido, la herramienta presentada en este trabajo quizá pueda utilizarse para el desarrollo de nuevas herramientas que faciliten el análisis de los emplazamientos de producto en otros medios como los medios impresos.

\section{Referencias bibliográficas}

Allen, R. C. (1985): Speaking of Soap Operas, Nueva York, Schoken Books.

AuTY, S.; LEWIS, C. (2004): «Exploring Children's Choice: The Reminder Effect of Product Placement», Psychology and Marketing, 21 (9), 697-708.

AVERY, R. J.; FERRARO, R. (2000): «Verisimilitude or advertising? Brand appearances on prime-time television», The Journal of Consumer Affairs, 34 (2), 217-244.

BABIN, L. A.; CARDER, S.T. (1996): «Viewers' Recognition of Brands Placed within a Film» International Journal of Advertising, 15 (2), 140-151.

Balasubramanian, S. K. (1994): «Beyond Advertising and Publicity: Hybrid Messages and Public Policy Issues», Journal of Advertising, 23 (4), 29-46.

Balasubramanian, S. K.; Karrh, J. A.; PAtwardhan, H. (2006): «Audience Response to Product Placements: An Integrative Framework and Future Research Agenda», Journal of Advertising, 35 (3), 115-141.

BAÑos, M.; RODRÍGUEZ, T. (2003): Product placement: estrella invitada la marca, Madrid, Cie Inversiones Editoriales Dossat 2000

BARDIN, L. (1977): El análisis de contenido, Madrid, Akal.

Beattie, A. E.; Mitchell A. A (1985): "The Relationship between Advertising Recall and Persuasion: An Experimental Investigation», en AlwitT, L. F. Y Mitchell, A. A (eds), Psychological Processes and Advertising Effects: Theory, Research, and Application, Hillsdale (Nueva Jersey), Erlbaum, 129-155. 
Berelson, B. (1952): Content Analysis in Communications Research. Glencoe (Illinois), The Free Press.

Brennan, I.; Dubas K. M.; Babin L. A. (1999): «The Influence of Product-Placement Type and Exposure Time on Product-Placement Recognition», International Journal of Advertising, 18 (3), 323-337.

CAuberghe, V.; De Pelsmacker, P. (2010): «Advergames: The Impact of Brand Prominence and Game Repetition on Brand Responses», Journal of Advertising, 39 (1), 5-18.

Chaney, I.; Lin, K.-H.; Chaney, J. (2004): «The Effect of Billboards within the Gaming Environment», Journal of Interactive Advertising, 5 (1), http://www.jiad.org/vol5/nol/chaney Web visitada el: 07/06/06.

Chang, C. (2002): «Self-Congruency as a Cue in Different Advertising-Processing Contexts», Communication Research, 29 (5), 503-536.

D’Astous, A.; SÉGuin, N. (1999): «Consumer Reactions to Product Placement Strategies in Television Sponsorship», European Journal of Marketing, 33 (10), 896-910.

D'Astous, A.; Chartier, F. (2000): «A Study of Factors Affecting Consumer Evaluations and Memory of Product Placements in Movies», Journal of Current Issues and Research in Advertising, 22 (2), 31-40.

De Gregorio, F.; Sung, Y. (2010): «Understanding Attitudes Toward And Behaviors In Response To Product Placement», Journal of Advertising, 39 (1), 83-96.

Delorme, D. E.; REID, L. N.; ZIMMER, M. R. (1994): «Brands in Films: Young Moviegoers' Experiences and Interpretations», en KING, K. W. (ed.), Proceedings of the 1994 Conference of the American Academy of Advertising. Athens (Georgia), American Academy of Advertising, 60 .

Del PINO, C. (2004): Marcas y ficción televisiva: el product placement en las teleseries españolas (1991-2002), Universidad de Málaga, Tesis doctoral.

Donaton, S. (2004): Madison \& Vine: Why the Entertainment and Advertising Industries Must Converge to Survive, Nueva York, McGraw-Hill.

FEARING, F. (1953): «Towards a Psychological Theory of Human Communication», Journal of Personality, 22, 71-88.

FERRARO, R.; AVERY, R. J. (2000): «Brand appearances on prime-time television», Journal of Current Issues and Research in Advertising, 22, 1-15.

Galician, M. L.; Bourdeau, P. G. (2004): «The Evolution of Product Placements in Hollywood Cinema: Embedding High-Involvement 'Heroic' Brand Images», Journal of Promotion Management, 10 (1/2), 15-36.

GATI, I.; TVERSKY, A. (1987): «Recall of Common and Distinctive Features of Verbal and Pictorial Stimuli», Memory \& Cognition, 15 (2), 97-100.

GLASS, Z. (2007): «The Effectiveness of Product Placement in Video Games», Journal of Interactive Advertising, 8 (1), http://jiad.org/article96 Web visitada el: 04/09/08.

GoldBerG, M. E.; GoRN, G. J. (1987): «Happy and Sad TV Programs: How They Affect Reactions to Commercials», Journal of Consumer Research, 14 (Diciembre), 387-403.

Gould, S. J.; GuPTA, P. B. (2006): «Come on Down': How Consumers View Game Shows and the Products Placed in Them», Journal of Advertising, 35 (1), 65-81.

Gould, S. J.; GuPTA, P. B.; GrABNER-KRÄUTER, S. (2000): «Product Placements in Movies: A Cross-Cultural Analysis of Austrian, French and American Consumer's Attitudes Toward This Emerging International Promotion Medium», Journal of Advertising, 29 (4), 41-58. 
Grigorovici, D. M.; Constantin, C. D. (2004): «Experiencing Interactive Advertising Beyond Rich Media: Impacts of Ad Type and Presence on Brand Effectiveness in 3D Gaming Immersive Virtual Environments», Journal of Interactive Advertising, 5 (1), http://jiad.org/vol5/nol/grigorovici/index.htm Web visitada el: 07/07/06.

GuptA, P. B.; Gould, S. J. (1997): «Consumer's Perceptions of the Ethics and Acceptability of Product Placement in Movies: Product Category and Individual Differences», Journal of Current Issues and Research in Advertising, 19 (1), 38-50.

GuPTA, P. B.; LORD, K. R. (1998): «Product Placement in Movies. The Effect of Prominence and Mode on Audience Recall», Journal of Current Issues and Research in Advertising, 20 (1), 47-59.

Hernández, M. D.; Chapa, S.; Minor, M. S.; Maldonado, C.; Barranzuela, F. (2004): «Hispanic Attitudes toward Advergames: A Proposed Model of their Antecedents», Journal of Interactive Advertising, 5 (1), http://jiad.org/vol5/no1/hernandez/index.htm Web visitada el: 05/08/06.

Howard, D. L.; BARRY, T. E. (1994): «The Role of Thematic Congruence Between a MoodInduced Event and an Advertised Product in Determining the Effects of Mood on Brand Attitudes», Journal of Consumer Psychology, 3 (1), 1-27.

Johnstone, E.; DodD, C. A. (2000): «Placements as mediators of brand salience within a U.K. cinema audience», Journal of Marketing Communications, 6 (3), 141-158.

KARRH, J. A. (1994): «Effects of Brand Placements in Feature Films», en KING, K. W. (ed.), Proceedings of the 1994 Conference of the American Academy of Advertising. Athens (Georgia), American Academy of Advertising, 90-96.

KASSARJIAN, H. H. (1977): «Content Analysis in Consumer Research», Journal of Consumer Research, 4 (1), 8-19.

KRIPPENDORF, K. (1980): Content analysis: and introduction to its methodology, Beverly Hills, Sage Publications, 46-51.

La Ferle, C.; Edwards, S. M. (2006): «Product Placement: How Brands Appear on Television», Journal of Advertising, 35 (4), 65-86.

LAW, S.; BRAUN, K. A. (2000): «I'll Have What She's Having: Gauging the Impact of Product Placements on Viewers», Psychology and Marketing, 17 (12), 1059-1075.

LEE, M.; FABER, R.J. (2007): «Effects of Product Placement in On-Line Games on Brand Memory», Journal of Advertising, 36 (4), 75-90

LEHU, J. M. (2007): Branded entertainment: product placement and brand strategy in the entertainment business, Londres y Filadelfia, Kogan Page.

LEWIS, B.; PORTER, L. (2010): «In-game advertising effects: examining player perceptions of advertising schema congruity in a massively multiplayer online role-playing game», Journal of Interactive Advertising, 10 (2), 46-60.

MallinCKRODT, V.; MizerSKI, D. (2007): «The Effects of Playing an Advergame on Young Children's Perceptions, Preferences, and Requests», Journal of Advertising, 36 (2), 87-100.

MAU, G.; SiLBERER, G.; CONSTIEN, C. (2008): «Communicating brands playfully: Effects of ingame advertising for familiar and unfamiliar brands», International Journal of Advertising, 27 (5), 827-851.

MARTí, J. (2010): Marketing y videojuegos: product placement, in-game advertising y advergaming, Madrid, ESIC.

MARTí, J.; MuÑOZ, P. (2008): Engagement Marketing: Una nueva publicidad para un marketing de compromiso, Madrid, Financial Times/Prentice Hall. 
MARTí, J.; AldÁs, J.; CuRRÁs, R.; SÁNCHEZ, I. (2010): «El emplazamiento de producto: conceptualización, nuevos formatos y efectos sobre el consumidor», Teoría y Praxis, 8, 113 136.

MCKeChnie, S. A.; ZHOU, J. (2003): «Product Placement In Movies: A Comparison Of Chinese And American Consumers' Attitudes», International Journal of Advertising, 22, 349-374.

MÉndiz, A. (2000): Nuevas Formas Publicitarias: Patrocino, Product Placement y Publicidad en Internet, Málaga, Servicio de Publicaciones de la Universidad de Málaga.

MOLESWORTH, M. (2006): «Real Brands in Imaginary Worlds: Investigating Players'Experiences of Brand Placement in Digital Games», Journal of Consumer Behaviour, 5 (4), 355-366.

Moore, E. S. (2006): «It's Child Play: Advergaming and the Online Marketing of Food to Children», The Henry J. Kaiser Family Foundation, http://www.kff.org/entmedia/upload/It-s-Child-s-Play-Advergaming-and-the-OnlineMarketing-of-Food-to-Children-Bios.pdf Web visitada el: 28/06/07.

Morton, C. R.; Friedman, M. (2002): «I Saw It in the Movies': Exploring the Link Between Product Placement Beliefs and Reported Usage Behaviour», Journal of Current Issues and Research in Advertising, 24 (2), 33-40.

NAIRNE, J. S.; NeATH, I.; SerRA, M.; ByUn, E. (1997): «Positional Distinctiveness and Ratio Rule in Free Recall», Journal of Memory \& Language, 37 (3), 155-166.

NeBEnZAHL, I. D.; SECUNDA, E. (1993): «Consumers' attitudes toward product placement in movies», International Journal of Advertising, 12 (1), 1-11.

Nelson, M. R.; KeuM, H.; YARos, R. A. (2004): «Advertainment or Adcreep? Game Players' Attitudes toward Advertising and Product Placements in Computer Games», Journal of Interactive Advertising, 5 (1), http:/www.jiad.org/vol5/nol/nelson/ Web visitada el: 18/06/06.

Nelson, M. R. (2002): «Recall of Brand Placements in Computer/Video Games», Journal of Advertising Research, 42 (2), 80-92.

Nelson, M. R.; YAros, R. A.; KeUM, H. (2006): «Examining The Influence Of Telepresence On Spectator And Player Processing Of Real and Fictitious Brands in a Computer Game», Journal of Advertising; 35 (4), 87-99.

Newell, J.; SAlmon, C. T.; Chang, S. (2006): «The Hidden History of Product Placement», Journal of Broadcasting and Electronic Media, 50 (4), 575-594.

NicOVICH, S. G. (2005): "The Effect of Involvement on Ad Judgment in a Video Game Environment: The Mediating Role of Presence», Journal of Interactive Advertising, 6 (1), http://www.jiad.org/vol6/nol/nicovich/index.htm Web visitada el: 03/08/07.

PAISLEY, W. J. (1969): «Studying Style as Deviation of Encoding Norm», en GERBNER, G. et alt. (eds.). The Analysis of Communications Content: Developments in Scientific Theories and Computer Techniques, Nueva York, Willey, 133-146.

PAIVIO, A. (1983): «The Empirical Case for Dual Coding» en Yuille, J. C. (ed). Imagery Memory and Cognition: Essays in Honour of Allan Paivio, Hillsdale (Nueva Jersey), Lawrence Erlbaum, 297-319.

PAIVIO, A. (1986): Mental Representations: A Dual Coding Approach, Nueva York, Oxford University Press.

PANDA, T. K. (2004): «Effectiveness of Product Placements in Indian Films and its effects on Brand Memory and Attitude with Special Reference to Hindi Films», The ICFAI Journal of Marketing Management, (Agosto), 42-56. 
PetTy, R. E.; CACIOPPO, J. T. (1986): «The Elaboration Likelihood Model of Persuasion», en Berkowitz, L. (ed.), Advances in Experimental Social Psychology, vol. 19, Nueva York, Academia Press, 123-205.

Petty, R. E.; Cacioppo, J. T.; Schumann, D. (1983): «Central and Peripheral Routes to Advertising Effectiveness: The Moderating Role of Involvement», Journal of Consumer Research, 10 (2), 135-146.

PQ MEDiA (2010): Global Branded Entertainment Marketing Forecast 2010-2014. En http://www.pqmedia.com/brandedentertainmentforecast2010.html Web visitada el: 07/08/10.

RUSSELL, C. A. (2002): «Investigating the Effectiveness of Product Placements in Televisión Shows: The Role of Modality and Plot Connection Congruence on Brand Memory and Attitude», Journal of Consumer Research, 29 (3), 306-318.

Russell, C. A.; STERN, B. B. (2006): «Consumers, Characters, and Products: A Balance Model of Sitcom Product Placement Effects», Journal of Advertising, 35 (1), 7-21.

Sabherwal, S.; POKRYwCZYNSKI, J.; Griffin, R. (1994): «Brand Recall for Product Placements in Motion Pictures: A Memory-Based Perspective», paper presentado en la Conference of the Association for Education in Journalism and Mass Communication, Atlanta (Georgia).

SAPOLSKY, B. S.; KINNEY, L. (1994): «You Oughta Be in Pictures: Product Placements in the Top Grossing Films of 1991», en KING, K. W. (ed.), Proceedings of the 1994 Conference of the American Academy of Advertising. Athens (Georgia), American Academy of Advertising, 89.

Sargent, J. D.; Tickle, J. J.; Beach, M. L.; Dalton, M. A.; Ahrens, M B.; Heatherton, T. F. (2001): «Brand appearances in contemporary cinema films and contribution to global marketing of cigarettes», Lacnet, 357, 29-32.

SCHMOLL, N.M.; HAFER, J.; HILT, M.; REILly, H. (2006): «Baby boomers' attitudes towards product placements», Journal of Current Issues and research in Advertising. 2 (8), 33-53.

SCHNEIDER, L. P.; CORNwELL, B. (2005): «Cashing in on crashes via brand placement in computer games: The effects of experience and flow on memory», International Journal of Advertising, 24, (3), 321-343.

Sengupta, J.; Goodstein, R. C.; Boninger, D. S. (1997): «All Cues Are Not Created Equal: Obtaining Attitude Persistent under Low-Involvement Conditions», Journal of Consumer Research, 23 (marzo), 351-361.

Spiegelman, M.; Terwilliger, C.; Fearing, F. (1953): «The Content of Comics: Goals and Means to Goals of Comic Strip Characters»,, Journal of Social Psychology, 37, 189-203.

SteOrTZ, E. M. (1987): The Cost Efficiency and Communication Effects Associated with Brand Name Exposure within Motion Pictures, Tesis doctoral, West Virginia University.

TAPIA, A.; LÓPEZ, M.; GONZÁLEZ, P. (2009): «Publicidad in-Game. Los videojuegos como soporte publicitario», Pensar la Publicidad. Revista Internacional de Investigaciones Publicitarias, 3 (2), 73-88.

TAYLOR, S. E.; THOMPSON, S. C. (1982): «Stalking the Elusive Vividness Effect», Psychological Review, 89 (2), 155-81.

Troup, M. M. (1991): The Captive Audience: A Content Analysis Of Product Placements In Motion Pictures, Tesis doctoral, Tallahassee, Florida State University.

ViCTORIA, J. S. (1999): Presencia, comunicación y estrategia de las marcas comerciales en la ficción audiovisual, Universidad de Málaga, Tesis doctoral 
VOLLMERS, S. M. (1995): The impact on children of brand and product placements in films, Tesis doctoral, Tallahassee, Florida State University.

VOLLMERS, S. M.; MiZERSKI, R. W. (1994): «A Review and Investigation into the Effectiveness of Product Placement in Films», en KING, K. W. (ed.). Proceedings of the 1994 Conference of the American Academy of Advertising. Athens (Georgia), American Academy of Advertising, 97-102.

WINKLER, T.; BUCKNER, K. (2006): «Receptiveness of Gamers to Embedded Brand Messages in Advergames: Attitudes towards Product Placement», Journal of Interactive Advertising, 7 (1), http://www.jiad.org/vol7/no1/winkler/index.htm Web visitada el: 07/04/07.

Wise, K.; Bolls, P. D.; Kim, H.; Venkataraman, A.; Meyer, R. (2008): «Enjoyment of Advergames and Brand Attitudes: The Impact of Thematic Relevance», Journal of Interactive Advertising, 9 (1), En http://www.jiad.org/article107 Web visitada el: 02/08/09.

YANG, M.; Roskos-EwOldSEN, D. R.; DINU, L.; ARPAN, L. M. (2006): «The Effectiveness of 'in-game' advertising: Comparing Collage Students' Explicit and Implicit Memory for Brand Names», Journal of Advertising, 35 (4), 143-152.

YI, Y. (1990): «The Effects of Contextual Priming in Print Advertisements», Journal of Consumer Research, 17 (2), p. 215-222.

ZIMMER, D. E.; ZIMMER, M. R. (1997): «The Effects of Brand Placement Type and a Disclaimer on Memory for Brand Placements in Movies», paper presentado en la Conference of the Association for Education in Journalism and Mass Communication, Chicago. 